\title{
A COUNTEREXAMPLE TO A CONJECTURE OF A. H. STONE
}

\author{
HAROLD BELL AND R. F. DICKMAN, JR.
}

\begin{abstract}
A. H. Stone has offered a sequence, $\{S(n) ; n>2\}$, of conjectures characterizing multicoherence for locally connected, connected, normal spaces. The conjecture $S(n)$ is, " $X$ is multicoherent if and only if $X$ can be represented as the union of a circular chain of continua containing exactly $n$ elements". It is known that $S(3)$ always obtains and that $S(6)$ obtains if the space is compact. In this paper, we construct a multicoherent plane Peano continuum $C$ for which $S(7)$ fails. Since $S(n+1)$ implies $S(n)$, $n>2, S(n)$ fails for $C$ for all $n>6$. Furthermore we show that for any integer $n>3$ there exists a plane Peano continuum for which $S(2 n)$ obtains while $S(2 n+1)$ fails.
\end{abstract}

Introduction. Throughout this paper $X$ will denote a locally connected, connected normal space. By a continuum we mean a closed and connected (not necessarily compact) subset of $X$. For $A \subset X, b_{0}(A)$ denotes the number of components of $A$ less one (or $\infty$ if this number is infinite). The degree of multicoherence, $r(X)$, of $X$ is defined by

$r(X)=\sup \left\{b_{0}(H \cap K): X=H \cup K\right.$ and $H$ and $K$ are subcontinua of $\left.X\right\}$.

If $r(X)=0, X$ is said to be unicoherent and we say that $X$ is multicoherent otherwise. By a chain $\kappa$ in $X$ we mean a finite collection of subcontinua of $X$ that can be ordered $\kappa=\left\{K_{1}, K_{2}, \ldots, K_{n}\right\}$ so that $K_{i} \cap K_{j} \neq \varnothing$ if and only if $|i-j| \leqslant 1$. A circular chain in $X$ is a collection of subcontinua $\kappa$ such that no three members of $\kappa$ have a point in common and if $K \in \kappa$, then $\kappa-\{K\}$ is a chain in $X$. Let $n>2$ be an integer and let $S(n)$ denote the following statement:

$S(n): X$ is multicoherent if and only if $X$ can be represented as the union of a circular chain containing exactly $n$ elements.

In a private communication, A. H. Stone conjectured that $S(n)$ is true for all $n>2$ and he stated that he had established $S(n)$ for all $n>2$ whenever $0<r(X)<\infty$. A. D. Wallace established $S(3)$ for Peano continua in [4]. A. H. Stone announced $S(3)$ for locally connected normal spaces in [3] and in [2], the second author included a proof of $S(3)$ for such spaces. In [1], the second author showed that $S(4)$ obtains for a large class of spaces and in [2], he showed that $S(6)$ always obtains if $X$ is compact. The purpose of this note

Presented to the Society August 17, 1977; received by the editors June 14, 1977 and, in revised form, February 26, 1978.

AMS (MOS) subject classifications (1970). Primary 54F55; Secondary 54F25.

Key words and phrases. Multicoherence, circular chain of continua.

๑ American Mathematical Society 1978 
is to give an example of a multicoherent plane Peano continuum for which $S$ (7) fails.

LemMa $\theta$. Let $a$ and $b$ be distinct points in $X$ and suppose that $X-\{a, b\}=$ $R \cup P \cup Q$ where $R, P$ and $Q$ are pairwise disjoint open connected sets and $\bar{R} \cap \bar{P} \cap \bar{Q}=\{a, b\}$. If $\kappa$ is a circular chain in $X$ and $\cup \kappa=X$ and some $K \in \kappa$ lies entirely in $R$, then $(\bar{P} \cup \bar{Q})$ meets at most four elements of $\kappa$.

Proof. Notice that $\{a, b\}$ is the boundary of each of $P, Q$ and $R$. Let $K_{0} \subset R$ and $\left\{K_{1}, K_{2}, \ldots, K_{n}\right\}$ be a chain representation of $\kappa-\left\{K_{0}\right\}$. Let $S$ be the union of those $K \in \kappa$ that contain $a$, let $T$ be the union of those $K \in \kappa$ that contain $b$, and let $V$ be the union of those $K \in \kappa$ that contain neither $a$ or $b$. Clearly, $V$ has at most two components, one of which contains $K_{0}$ and is therefore contained in $R$. It follows that either $\bar{P}$ or $\bar{Q}$ must fail to intersect $V$. Therefore either $\bar{Q} \subset S \cup T$ or $\bar{P} \subset S \cup T$. Since both $P$ and $Q$ are connected and intersect both $S$ and $T$ it follows that $S \cap T \neq \varnothing$. That is, there is a $k \geqslant 0$ such that $\{a, b\} \subset K_{k} \cup K_{k+1}$. It follows that $K_{i} \subset R$ except possibly when $i=k-1, k, k+1$ or $k+2$.

Construction of the example. For each positive integer $i$, let $C_{i}$ be a one-dimensional simplicial complex in the plane, with $V_{i}$ as its set of vertices and $\mathcal{E}_{i}$ as its set of edges, constructed as follows:

Let $V_{1}$ consist of three evenly distributed points on the unit circle $\{z$ : $|z|=1\}$ and let $\varepsilon_{1}$ consist of three connecting open intervals. Then $C_{1}=$ $\cup\left\{I: I \in \mathcal{E}_{1}\right\} \cup V_{1}$. Let $\mathscr{Q}_{1}=\left\{U(I): I \in \mathcal{E}_{1}\right\}$ be a set of mutually disjoint bounded open convex sets such that $I \subset U(I)$ for $I \in \mathcal{E}_{1}$. Suppose $C_{n}, V_{n}, \mathcal{Q}_{n}$, and $\mathcal{E}_{n}$ have been defined. For each $I \in \mathcal{E}_{n}$ let $a(I), b(I)$ be the endpoints of $I$, and $m(I)$ its midpoint $(a(I)+b(I)) / 2$. Let $t_{n}$ be chosen so that $1<t_{n}<1+1 / n$ and (for all $I \in \mathcal{E}_{n}$ ) the two "half-open" line segments $\left(a(I), t_{n} m(I)\right],\left[t_{n} m(I), b(I)\right)$ are both contained in $U(I)$. Let $V_{n+1}=V_{n} \cup\left\{m(I): I \in \mathcal{E}_{n}\right\} \cup\left\{t_{n} m(I): I \in \mathcal{E}_{n}\right\}$. Let $\mathcal{E}_{n+1}=\{(a(I)$, $\left.m(I)): I \in \mathcal{E}_{n}\right\} \cup\left\{(m(I), b(I)): I \in \mathcal{E}_{n}\right\} \cup\left\{\left(a(I), t_{n} m(I)\right): I \in \mathcal{E}_{n}\right\} \cup$ $\left\{\left(t_{n} m(I), b(I)\right): I \in \mathcal{E}_{n}\right\}$. Then $C_{n+1}=\cup\left\{I: I \in \mathcal{E}_{n+1}\right\} \cup V_{n+1}$. Let $\mathscr{Q}_{n+1}$ $=\left\{U(I): I \in \mathcal{E}_{n+1}\right\}$ be a set of mutually disjoint open convex sets such that if $I \in \mathscr{Q}_{n+1}$ and $J \in \mathscr{Q}_{n}$ and $I \subset U(J)$ then $I \subset U(I) \subset U(J)$. Let $D=\cup_{i=1}^{\infty} C_{i}$ and let $C=\bar{D}$.

It is clear that $C$ is multicoherent. By Theorems 3 and 6 of [2], $S(6)$ obtains for $C$. We will now show that $S(7)$ fails for $C$. (Since $(S n+1)$ always implies $S(n), S(k)$ for $k>6$ fails for $C$.)

DeFINITION. For $I=(a(I), b(I)) \in \mathcal{E}_{n}$ let $I^{\prime}=\left(a(I), t_{n} m(I)\right] \cup\left[t_{n} m(I)\right.$, $b(I))$; we use the convention $\left(I^{\prime}\right)^{\prime}=I$. Let $\mathcal{E}_{n}^{\prime}=\left\{I^{\prime}: I \in \mathcal{E}_{n}\right\} \cup \mathcal{E}_{n}$. The following lemma seems clear.

Lemma A. (1) $C$ is a Peano continuum.

(2) If $I \in \mathcal{E}_{n}^{\prime}$ has endpoints $a(I)$ and $b(I)$ then $C-\{a(I), b(I)\}$ has exactly three components $P(I), P\left(I^{\prime}\right)$ and $Q(I)$ where $I \subset P(I)$ and $I^{\prime} \subset P\left(I^{\prime}\right)$.

(3) If, for each $i, I_{i} \in \mathcal{E}_{i}^{\prime}$ then $\lim _{i \rightarrow \infty} \operatorname{dia}\left(I_{i}\right)=\lim _{i \rightarrow \infty} \operatorname{dia} P\left(I_{i}\right)=0$. 


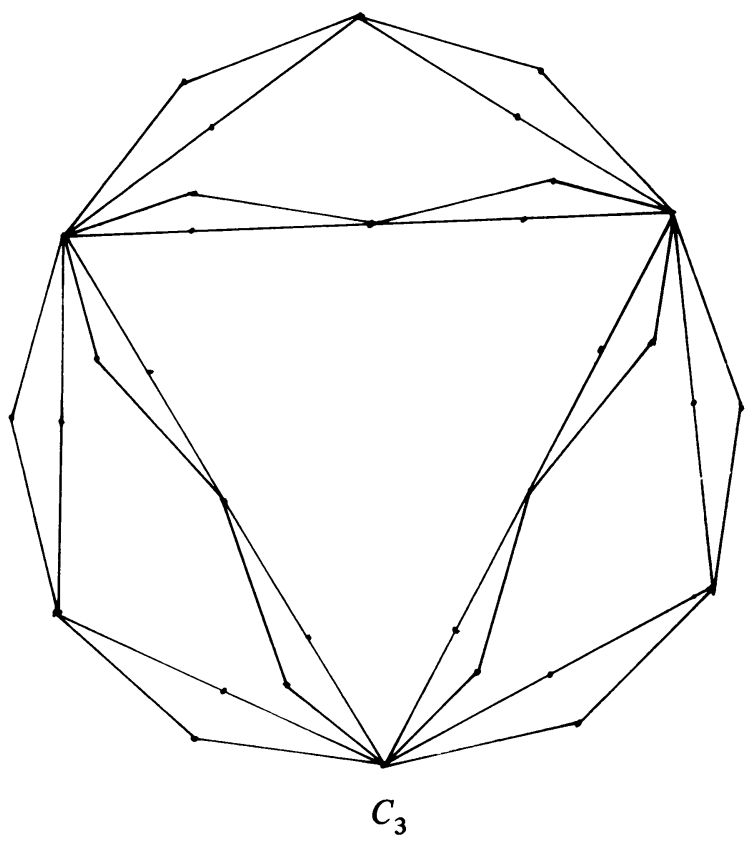

THEOREM 1. The plane Peano continuum $C$ cannot be the union of a circular chain with seven elements.

Proof. Suppose to the contrary that $\kappa$ is a circular chain with seven elements and $\cup \kappa=C$. Since each of the three vertices of $C_{1}$ is contained in at most two $K \in \kappa$ it follows that some $K \in \kappa$ contains no vertex of $C_{1}$ and is therefore contained in $P\left(I_{1}\right)$ for some $I_{1} \in \mathcal{E}_{1}^{\prime}$. If $a$ and $b$ are the endpoints of $I_{1}$, we may write $C-\{a, b\}$ as the union of $R=P\left(I_{1}\right), P=P\left(I_{1}^{\prime}\right)$ and $Q=Q\left(I_{1}\right)$. According to Lemma $\theta,(\bar{P} \cup \bar{Q})$ meet at most four elements of $\kappa$ and so $R$ contains three elements, say $K_{1}^{1}, K_{2}^{1}$, and $K_{3}^{1}$. Now the vertex of $C_{2}$ that lies in $R$, must miss one of these $K_{i}^{1}$ 's, say $K_{1}^{1}$. Then there is an $I_{2} \in \mathcal{E}_{2}^{\prime}$ such that $P\left(I_{2}\right)$ contains $K_{1}^{1}$. Let $a_{2}, b_{2}$ be the endpoints of $I_{2}$ and note that

$$
C-\left\{a_{2}, b_{2}\right\}=P\left(I_{2}\right) \cup P\left(I_{2}^{\prime}\right) \cup Q\left(I_{2}\right) \text {. }
$$

Again by Lemma $\theta, P\left(I_{2}\right)$ must contain 3 elements of $\kappa$ and hence the new vertex of $C_{3}$ that lies in $P\left(I_{2}\right)$ must miss one of these elements of $\kappa$. We continue as above and select a sequence $I_{2}, I_{3}, \ldots$, such that $I_{j} \in \mathcal{E}_{j}^{\prime}$, $P\left(I_{(j+1)}\right) \subset P\left(I_{j}\right)$ and each $P\left(I_{j}\right)$ contains a member of $\kappa, K_{j}$. Since $\kappa$ is finite, there is a subsequence $\left\{I_{j_{k}}\right\}$ such that $K_{j_{s}}=K_{j_{r}}$ for all $r, s \geqslant 1$. But then $K_{j_{1}} \subset \cap_{n=2}^{\infty} P\left(I_{n}\right)$, and, by Lemma A, $K_{j_{1}}$ must be a singleton. Of course, this is impossible and this completes the proof.

Theorem 2. For all integers $n \geqslant 3$, there exists a plane Peano continuum $P(n)$ such that $S(2 n)$ obtains but $S(2 n+1)$ fails.

Proof. We construct $P(n)$ in the same fashion we constructed $C$ except in this instance we change the example by letting $C_{1}$ be a regular $n$-gon. If $I$ is an edge in $C_{1}$ with endpoints $a$ and $b$ then $\overline{P(I)} \cup \overline{P\left(I^{\prime}\right)}$ can be easily written 
as the union of two continua $K_{I}^{a}$ and $K_{I}^{b}$ where $a \notin K_{I}^{b}$ and $b \notin K_{I}^{a}$. Then $\left\{K_{I}^{a}: I\right.$ is an edge in $\left.C_{1}\right\} \cup\left\{K_{I}^{b}: I\right.$ is an edge in $\left.C_{1}\right\}$ is a circular chain that covers $P(n)$ and has $2 n$ elements. Let $\kappa$ be any circular chain that covers $P(n)$. If every element of $\kappa$ contains a vertex of $C_{1}$ then $\kappa$ has at most $2 n$ elements. If some element of $\kappa$ fails to contain a vertex of $C_{1}$ then there is an $I \in \mathcal{E}_{1}^{\prime}$ such that $P(I)$ contains an element of $\kappa$. The same proof used for $P(3)$ then shows that in this case $\kappa$ has at most 6 elements.

REMARK. The authors have not been able to construct an example of a plane Peano continuum $P$ for which $S(2 n+1)$ obtains while $S(2 n+2)$ fails.

\section{REFERENCES}

1. R. F. Dickman, Jr., Some mapping characterizations of unicoherence, Fund. Math. 78 (1973), $27-35$.

2. _ Multicoherent spaces, Fund. Math. 91 (1976), 219-229.

3. A. H. Stone, Incidence relations in unicoherent spaces, Trans. Amer. Math. Soc. 65 (1949), 427-447.

4. A. D. Wallace, A characterization of unicoherence, Bull. Amer. Math. Soc. 48 (1952), 445.

Department of Mathematics, University of Cincinnati, Cincinnati, Oho 45221

Department of Mathematics, Virginia Polytechnic Institute and State University, BLACKSBURG, Virginia 24061 